\title{
Static and Dynamic Dielectric Properties of Mesogenic $n$-Nonyloxycyanobiphenyl (9OCB)
}

\author{
Grzegorz Czechowski and Jan Jadżyn \\ Institute of Molecular Physics, Polish Academy of Sciences, M. Smoluchowskiego 17, \\ 60-179 Poznań, Poland \\ Reprint requests to Prof. J. J.; E-mail: jadzyn@ifmpan.poznan.pl
}

Z. Naturforsch. 62a, 61 - 66 (2007); received October 30, 2006

\begin{abstract}
The dielectric properties of $n$-nonyloxycyanobiphenyl in the isotropic (I), nematic (N) and smectic $A\left(S_{A}\right)$ phases were investigated. The dielectric relaxation spectra, recorded in the frequency range $50 \mathrm{kHz}-100 \mathrm{MHz}$, were analyzed with use of the Cole-Cole equation. An anomalous temperature behavior of the static permittivity, the rotational diffusion exponent and the activation energy of mesogenic molecules rotating around their short axis, observed in the vicinity of the phase transitions, are discussed.
\end{abstract}

Key words: $n$-Nonyloxycyanobiphenyl; 9OCB; Dielectric Properties; Relaxation; Anomalous Rotational Diffusion Exponent; Activation Energy.

\section{Introduction}

Phase transitions from isotropic (I) liquid to nematic $(\mathrm{N})$ liquid crystals or between different liquid crystalline mesophases are spectacular and remarkable macroscopic events in nature. These transitions are either weakly discontinuous or continuous. They usually display a critical-like behavior mainly due to strong fluctuational effects. In case of the I-N transition one measures a small jump in the molar volume and a latent heat of about $10 \%$ of those observed for the first order process of crystallization, so the transition is classified as of weakly first order or close to second order. Consequently, in the vicinity of the I-N transition one observes a critical-like behavior of many physical properties. The experimental results are mostly interpreted by the de Gennes theory [1], which adopts the Landau theory of a second order phase transition to a weakly first order one. The validity of the Landau-de Gennes theory has been experimentally confirmed by static measurements of magnetic [2] and electric [3] birefringence, light scattering [4], and the nonlinear dielectric effect [5], as well as by dynamic studies of molecular spin-lattice relaxation $[6,7]$, dynamic light scattering [8], the transient grating optical Kerr effect $[9,10]$, and nonlinear dielectric relaxation $[11,12]$. In case of the nematic to smectic $\mathrm{A}\left(\mathrm{S}_{\mathrm{A}}\right)$ transition, the most spectacular pretransitional effects concern the viscous [13-16] and elastic properties [17-20] of nematic liquid crystals.

In the linear dielectric properties the prenematic effects are observed for strongly polar isotropic liquids as a peculiar temperature dependence of the static permittivity [21-25], the activation energy for molecular rotation around their short axis [23-28] or as an anomalously slow rotational diffusion, which manifests itself as a broadening of the dielectric absorption spectra $[26,28]$.

In this paper we analyze the pretransitional effects observed in the static and dynamic dielectric properties of the mesogenic compound $n$-nonyloxycyanobiphenyl (9OCB) with a relatively narrow temperature range of the nematic phase existence between the isotropic liquid and the smectic A phase.

\section{Experimental}

$n$-Nonyloxycyanobiphenyl $\left(\mathrm{C}_{9} \mathrm{H}_{19} \mathrm{OPhPhCN}, 9 \mathrm{OCB}\right)$, with the following sequence of phase transitions: (Cr) $65{ }^{\circ} \mathrm{C},\left(\mathrm{S}_{\mathrm{A}}\right) 76.1{ }^{\circ} \mathrm{C}$, (N) $80.2{ }^{\circ} \mathrm{C}(\mathrm{I})$, was synthesized and purified at the Institute of Chemistry, Military University of Technology, Warsaw. Dielectric properties of the compound were measured with a HP 4194A impedance/gain phase analyzer in the frequency region from $50 \mathrm{kHz}$ to $100 \mathrm{MHz}$. The static values of the permittivity were taken as a low-frequency 
plateau of the real part of the complex dielectric permittivity. The measuring capacitor consisted of three plane electrodes: one central and two grounded on each side. In the nematic and smectic A phases a dc biasing electric field was applied to the capacitor, so the parallel component of the permittivity $\varepsilon_{\|}^{*}(\boldsymbol{n} \| \boldsymbol{E})$ was measured. The electrical heating of high performance, obtained by using a Unipan 650 controller, assured a quite good stabilization of the temperature (better than $10^{-3} \mathrm{~K}$ ) with the possibility of varying the temperature by steps of $10^{-2} \mathrm{~K}$. Such equipment allowed us to determine the permittivity with a relative accuracy better than $0.5 \%$.

\section{Results and Discussion}

Figure 1 presents the dielectric relaxation spectra [dispersion $\varepsilon^{\prime}(\omega)$ and absorption $\varepsilon^{\prime \prime}(\omega)$ ] measured in the isotropic, nematic and smectic A phases of 9OCB, in the temperature range of $60-90{ }^{\circ} \mathrm{C}$. For a probing electric field of low frequency, the real part of the permittivity $\left(\varepsilon^{\prime}\right)$ is frequency-independent (static dielectric constant, $\left.\varepsilon_{\text {stat }}\right)$ and its value reflects, at a given number of dipolar molecules per unit volume, the collective molecular contribution to the dielectric polarization of the sample studied. The main provenience of the contribution comes from the molecular orientation effects due to an external orienting field (as in the nematic and smectic A phases) or due to the intermolecular dipolar correlations (as in isotropic liquids). Figure 2 shows the temperature dependence of the static permittivity determined in the isotropic, nematic and smectic A phases of 9OCB. A strong increase of the permittivity, observed just after the transition to the nematic liquid crystalline phase, is a consequence of the above-mentioned collective orientation of 9OCB molecules due to the application of an external biasing electric field.

A detailed analysis of the static permittivity, measured in the isotropic phase of $90 \mathrm{CB}$, as presented in Fig. 3, shows the molecular behavior in a pretransitional region when the nematic phase approaches. Several degrees before the transition to the nematic phase, the static permittivity shows a maximum, as usually observed for strongly polar mesogenic liquids $[25,29,30]$. This pretransitional effect reflects a significant enhancement of the antiparallel dipolar aggregation in the isotropic liquid when the transition to the liquid crystalline nematic phase approaches. It is worth mentioning that the effect is not observed in case
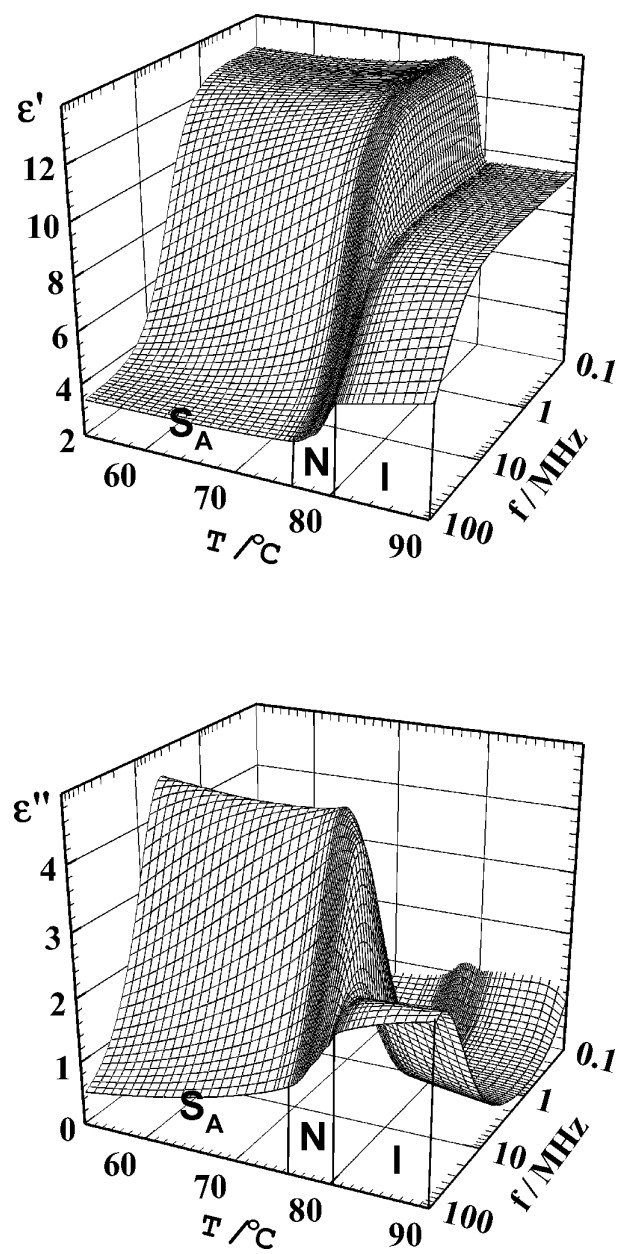

Fig. 1. Dielectric relaxation spectra of $90 \mathrm{CB}$ recorded in the isotropic $(\mathrm{I})$, nematic $(\mathrm{N})$ and smectic $A\left(\mathrm{~S}_{\mathrm{A}}\right)$ phases. In the nematic and smectic phases the permittivity $\varepsilon_{\|}^{*}(\omega, T)$ was measured.

of the first order phase transition from the isotropic liquid to the solid crystalline phase [24].

For mesogenic cyanobiphenyl molecules, like 9OCB, with a permanent dipole moment directed roughly along the long molecular axis, the temperature evolution of the static permittivity in the isotropic phase can be reproduced by the equation [25]

$$
\varepsilon_{\text {stat }}(T)=\varepsilon_{\text {extr }}+A\left(T-T^{*}\right)+B\left(T-T^{*}\right)^{1-\sigma},
$$

where $T^{*}$ is the extrapolated temperature of a virtual continuous phase transition, $\varepsilon_{\text {extr }}$ is the extrapolated value of the dielectric permittivity at $T^{*}, A$ and $B$ are amplitudes, and $\sigma$ is the exponent associated with a prenematic behavior. The result of the best fit of (1) to the experimental data is represented by the solid line 


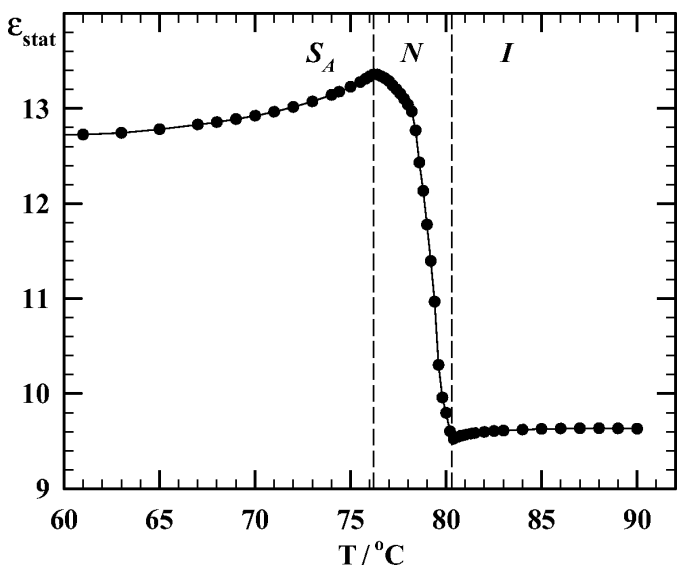

Fig. 2. Temperature dependence of the static dielectric permittivity of 9OCB. In the nematic and smectic A phases $\varepsilon_{\| \text {stat }}$ was measured.

in Fig. 3, and the resulting values of the main fitting parameters are shown in the inset of the figure. The obtained value of $\Delta T=T_{\mathrm{NI}}-T^{*}$, equal to $0.3^{\circ} \mathrm{C}$, shows the very weak discontinuous character of the I-N transition in 9OCB and the value of the critical exponent $\sigma=0.48$ corresponds to the tricritical nature of this transition [30,31].

The dielectric spectra presented in Fig. 1 were resolved into two elementary contributions of the ColeCole type [32]:

$$
\begin{aligned}
\varepsilon^{*}(\omega, T) & =\varepsilon^{\prime}(\omega, T)-\mathrm{i} \varepsilon^{\prime \prime}(\omega, T) \\
& =\varepsilon_{\infty}(T)+\sum_{k=1}^{2} \frac{A_{k}(T)}{1+\left[\mathrm{i} \omega \tau_{k}(T)\right]^{\alpha}},
\end{aligned}
$$

corresponding to molecular rotations around their two principal axes. In (2) $\omega$ denotes the angular frequency, $\varepsilon_{\infty}$ is the permittivity measured at a sufficiently high frequency to prevent dipolar reorientation, $A_{k}$ is the dielectric strength and $\tau_{k}$ is the relaxation time of the $k$-th relaxation process.

The strength of the dielectric absorption band, corresponding to the rotation of the 9OCB molecules around their long axis, is much smaller than that corresponding to the rotation around the short axis, and besides, its sensibility to the phase transitions is very weak, so we will not discuss this contribution here.

Figure 4 presents (in form of an Arrhenius plot) the temperature dependence of the dielectric relaxation time $(\tau)$, corresponding to the Brownian rotation of $90 C B$ molecules around their short axis. The dependence results from the best fit of (2) to the experimental

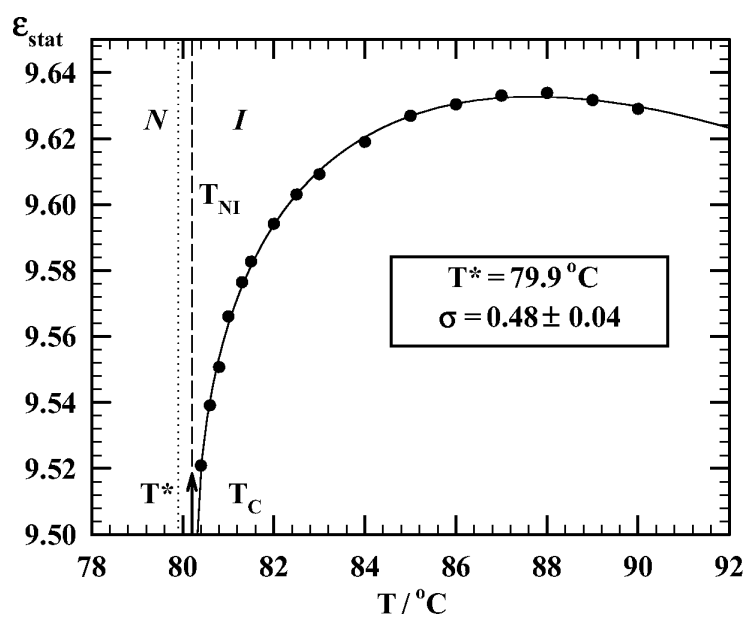

Fig. 3. Critical-like temperature dependence of the static permittivity of isotropic 9 OCB in the vicinity of the isotropic to nematic phase transition. The solid line represents the best fit of (1) to the experimental data (full circles).

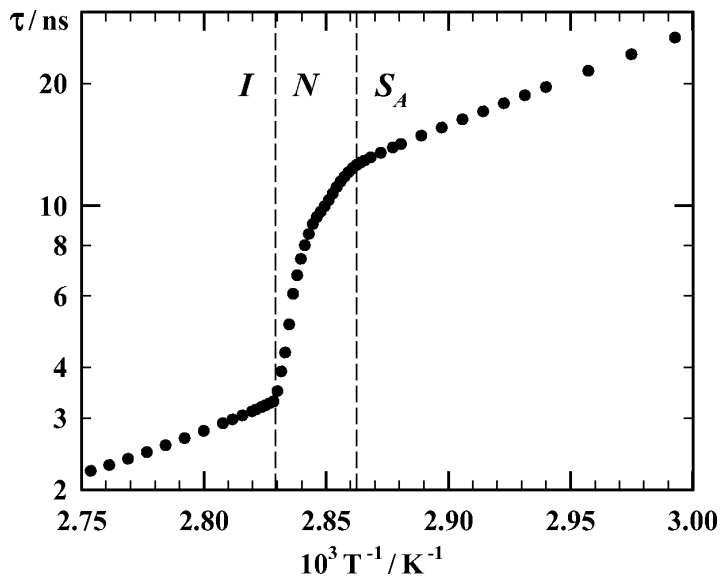

Fig. 4. Arrhenius plot for the relaxation time corresponding to the rotation of $9 \mathrm{OCB}$ molecules around their short axis.

spectra. Using the Arrhenius equation

$$
\tau(T)=C \exp \left(\frac{E_{\mathrm{A}}}{R T}\right)
$$

one assumes that the value of the relaxation time is determined by the ratio of the activation $\left(E_{\mathrm{A}}\right)$ and the thermal $(R T)$ energies. $C$ is a constant. However, as the meaning of the "activation energy" in molecular Brownian rotational motion is not clear, the postulate of a constancy of $E_{\mathrm{A}}$ as function of temperature, seems to have no physical basis. As the main physical properties of liquids are temperature-dependent, one should expect rather an increase of $E_{\mathrm{A}}$ with decreasing temperature, mainly due to an increase of 


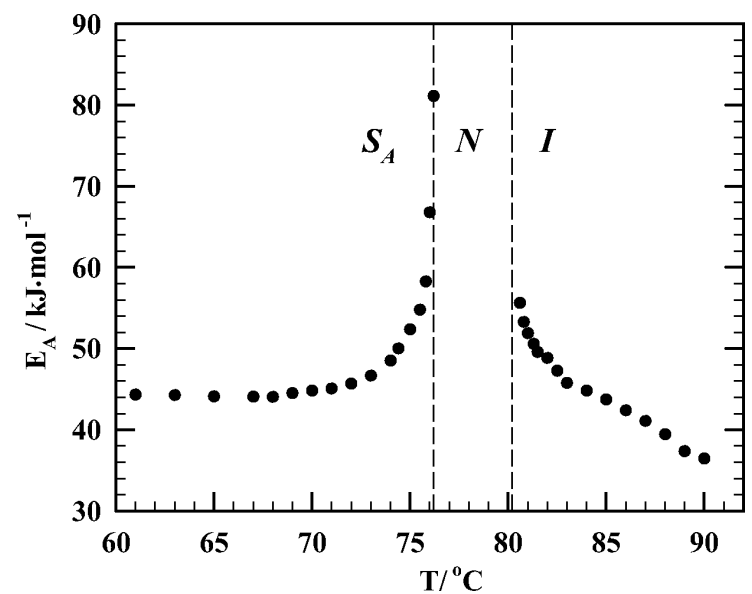

Fig. 5. Temperature dependence of the activation energy of 9OCB molecules in diffusional rotation around their short axis in the vicinity of $\mathrm{I}-\mathrm{N}$ and $\mathrm{S}_{\mathrm{A}}-\mathrm{N}$ phase transitions.

the molecules number per unit volume. That is why we have proposed [23] a procedure for the determination of $E_{\mathrm{A}}$ from the differentiation of (3). The derivative $\mathrm{d}(\ln \tau) / \mathrm{d}\left(T^{-1}\right)$ leads directly to the activation energy at a given temperature, so allowing one to analyze how $E_{\mathrm{A}}$ evolves with $T$. Figure 5 presents the temperature dependence of the activation energy obtained in that way. Indeed, far from the phase transitions, both in the smectic phase and in the isotropic liquid, one observes an increase of the activation energy with decreasing temperature; the effect is essentially more pronounced for the isotropic liquid. However, the method, consequently applied for the relaxation time of $9 \mathrm{OCB}$ in the whole temperature range studied, reveals an important temperature dependence of the activation energy in the vicinity of the phase transitions. An unusual strong temperature dependence of the relaxation time in the nematic phase of $90 \mathrm{OB}$, which can be seen in Fig. 4, leads to quite high values of $E_{\mathrm{A}}$, so they are not depicted in Figure 5. Because of the not clear physical meaning of $E_{\mathrm{A}}$, its temperature dependence probably reflects an unusual molecular dynamics in the vicinity of the phase transitions in comparison to that far from the transitions. One must stress that the observed continuous changes of the activation energy are surely a consequence of the pre- and posttransitional transformations on the molecular level. However, the discussion requires here a theoretical model describing the relation between the dielectric relaxation time/temperature behavior and a type of the molecular diffusion processes occurring in the liquid studied. The experimental re-

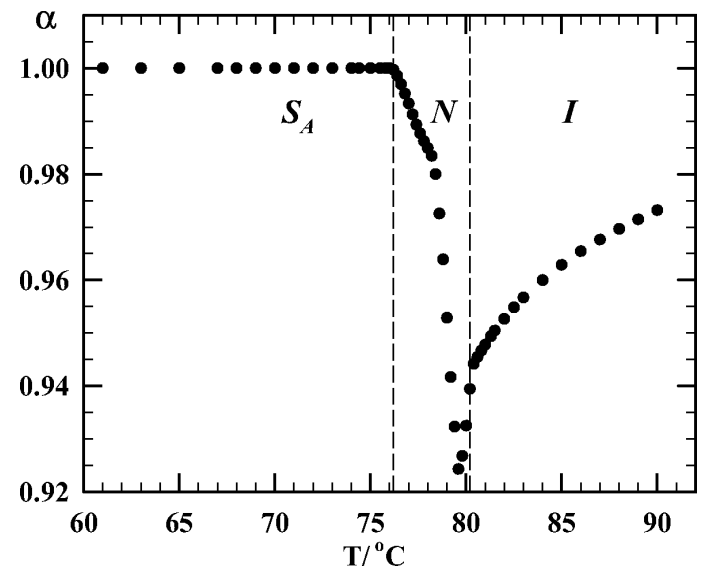

Fig. 6. Temperature dependence of the anomalous rotational diffusion exponent $\alpha$ obtained from the best fit of the Cole-Cole equation (2) to the experimental dielectric spectra of $9 \mathrm{OCB}$.

sults presented below suggest the existence of such relation.

As shown in many theoretical papers [33-41], an anomaly in the Brownian rotational diffusion of molecules leads to some changes in the shape of the dielectric absorption band. The subdiffusion, in its simplest case, leads to a symmetric broadening of the band, in comparison to that predicted by the Debye equation. Experimental results are then well described by the Cole-Cole equation (2), which was previously considered as an empirical one and only quite recently [34-37] has received a strong physical background. It was found that the Cole-Cole relaxation behavior is a direct consequence of an anomalously slow molecular rotational diffusion, and the exponent $\alpha$ in (2) reflects the extent of that anomaly. For the normal Brownian diffusion, which leads to a simple exponential decay of the dielectric response in the time domain and to the Debye equation for the complex permittivity in the frequency domain, the exponent $\alpha$ is equal to 1 . In case of the subdiffusion, the exponent $\alpha<1$ and the relaxation processes in a system can no more be described by the normal Brownian dynamics of the random walks with an exponential decay

$$
f(t)=\exp \left(-\frac{t}{\tau}\right)
$$

where $f(t)$ is the normalized relaxation function, but by the continuous-time random walk with a power-law distribution of the waiting times between the consecutive diffusional jumps of the rotating molecules. In the case of the fractional value of $\alpha$, the exponential way 
of equilibration of time-dependent quantities must be substituted by the Mittag-Leffler pattern [42] that interpolates between an initial stretched exponential and a terminal inverse power-law pattern, both of index $\alpha$ :

$$
E_{\alpha}\left[-(t / \tau)^{\alpha}\right] \propto\left\{\begin{array}{l}
\exp \left[-\frac{(t / \tau)^{\alpha}}{\Gamma(1+\alpha)}\right], t \ll \tau \\
{\left[\Gamma(1-\alpha)(t / \tau)^{\alpha}\right]^{-1}, t \gg \tau}
\end{array}\right.
$$

The Mittag-Leffler function is the exact relaxation function leading directly to the Cole-Cole equation (2). In the limit of $\alpha=1$, the Mittag-Leffler function reduces itself to the exponential function given by (4).

Figure 6 presents the temperature dependence of the anomalous rotational diffusion exponent $\alpha$ resulting from the best fit of the Cole-Cole equation (2) to the experimental dielectric spectra of 9OCB. Far from the phase transitions, both in the isotropic and smectic A phases of the compound, the value of the exponent $\alpha$

[1] P. G. de Gennes, Physics of Liquid Crystals, Oxford University Press, Oxford 1974.

[2] J. D. Litster and T. W. Stinson III, J. Appl. Phys. 41, 996 (1970).

[3] J. C. Filippini and Y. Poggi, Phys. Lett. 65A, 30 (1978).

[4] T.D. Gierke and W.H. Flygare, J. Chem. Phys. 61, 2231 (1974).

[5] A. Drozd-Rzoska, S. J. Rzoska, and J. Zioło, Phys. Rev. E 54, 6452 (1996).

[6] B. Cabane and G. Clarke, Phys. Rev. Lett. 25, 91 (1970).

[7] S. Gosh, E. Tettamanti, and E. Indovina, Phys. Rev. Lett. 29, 638 (1973).

[8] T. W. Stinson III and J. D. Litster, Phys. Rev. Lett. 25, 503 (1970).

[9] F. W. Deeg, S. R. Greenfield, J. J. Stankus, V. J. Newell, and M. D. Fayer, J. Chem. Phys. 93, 3503 (1990).

[10] J. J. Stankus, R. Torre, C. D. Marshall, S. R. Greenfield, A. Sengupta, A. Tokmakoff, and M. D. Fayer, Chem. Phys. Lett. 194, 213 (1992).

[11] P. Kȩdziora, J. Jadżyn, and L. Hellemans, Phys. Rev. E 66, 021709, 031702 (2002).

[12] J. Jadżyn, P. Kedziora, and L. Hellemans, in: Relaxation Phenomena in Dielectric, Magnetic and Superconducting Materials (Eds. W. Haase and S. Wróbel), Springer-Verlag, Heidelberg 2003, pp. 51-71.

[13] H.-H. Graf, H. Kneppe, and F. Schneider, Mol. Phys. 77, 521 (1992).

[14] J. Jadżyn and G. Czechowski, J. Phys.: Condens. Matter 13, 261 (2001). is close to unity, indicating a normal Brownian rotational diffusion of the molecules. The effect is more pronounced in the smectic phase, where the dielectric absorption bands show a quite perfect Debye's shape $(\alpha \approx 1)$. A similar behavior was observed in case of $n$-nonylcyanobiphenyl (9CB) [43]. Also similarly to $9 \mathrm{CB}$, due to a relatively narrow temperature range of the nematic phase of $90 \mathrm{OCB}\left(4^{\circ} \mathrm{C}\right)$, an anomalous decrease of the exponent $\alpha$ is observed not only in the vicinity of the phase transitions, but extents into the whole range of the existence of the nematic phase in the compound. Temperature dependences of the anomalous rotational diffusion exponent $\alpha$ and the activation energy $E_{\mathrm{A}}$ exhibit so many similarities that they can suggest the same provenience of the anomalous behavior of these two quantities in the vicinity of the phase transitions where strong fluctuation effects are leading to the formation of pseudonematic domains in the isotropic liquid or pseudosmectic layers in the nematic phase.

[15] J. Jadżyn and G. Czechowski, Phys. Rev. E 64, 052702 (2001).

[16] J. Jadżyn and G. Czechowski, Acta Phys. Pol. A 105, 459 (2004).

[17] M. Simoes, A.J. Palangana, A. R. Sampaio, A. de Campos, and K. E. Yamaguti, Phys. Rev. E 71, 051706 (2005).

[18] P. E. Cladis, Phys. Rev. Lett. 31, 1200 (1973).

[19] M. J. Bradshaw, E. P. Raynes, J. D. Bunning, and T. E. Faber, J. Phys. 46, 1513 (1985).

[20] G. Czechowski and J. Jadżyn, Acta Phys. Pol. A 106, 475 (2004).

[21] A. Drozd-Rzoska, S. J. Rzoska, and J. Zioło, J. Phys.: Condens. Matter 12, 6135 (2000).

[22] S. J. Rzoska, A. Drozd-Rzoska, J. Zioło, P. Hajdas, and J. Jadżyn, Phys. Rev. E 64, 061104 (2001).

[23] J. Jadżyn, G. Czechowski, C. Legrand, and R. Douali, Phys. Rev. E 67, 041705 (2003).

[24] M. Ginovska, H. Kresse, D. Bauman, G. Czechowski, and J. Jadżyn, Phys. Rev. E 69, 022701 (2004).

[25] S. J. Rzoska, J. Ziolo, W. Sulkowski, J. Jadżyn, and G. Czechowski, Phys. Rev. E 64, 052701 (2001).

[26] J. Jadżyn, J.-L. Déjardin, G. Czechowski, and M. Ginovska, J. Phys.: Condens. Matter 17, 813 (2005).

[27] J. Jadżyn, G. Czechowski, and J.-L. Déjardin, J. Phys.: Condens. Matter 18, 1839 (2006).

[28] M. Ginovska, G. Czechowski, J.-L. Déjardin, J. Jadżyn, and L. Hellemans, Liq. Cryst. 32, 625, (2005). 
[29] A. Drozd-Rzoska, S. J. Rzoska, and J. Zioło, Phys. Rev. E 61, 5349 (2000).

[30] M. A. Anisimov, Critical Phenomena in Liquids and Liquid Crystals, Gordon and Breach, New York 1991.

[31] A. Żywociński, J. Phys. Chem. B 107, 9491 (2003).

[32] K. S. Cole and R.H. Cole. J. Chem. Phys. 9, 341 (1941).

[33] W. G. Glockle and T. I. Nonnenmacher, J. Stat. Phys. 71, 741 (1993)

[34] K. Weron and M. Kotulski, Physica A 232, 180 (1996).

[35] R. Metzler and J. Klafter, Adv. Chem. Phys. 116, 223 (2001).

[36] R. Hilfer, J. Non-Cryst. Solids 305, 122 (2002).
[37] R. Metzler and J. Klafter, J. Non-Cryst. Solids 305, 81 (2002).

[38] W. T. Coffey, Yu. P. Kalmykov, and S. V. Titov, J. Chem. Phys. 116, 6422 (2002).

[39] W. T. Coffey, Yu. P. Kalmykov, and S. V. Titov, Phys. Rev. E 65, 032102 (2002).

[40] J.-L. Déjardin, Phys. Rev. E 68, 031108 (2003).

[41] J.-L. Déjardin and J. Jadżyn, J. Chem. Phys. 122, 074502 (2005); 123, 174502 (2005).

[42] G. M. Mittag-Leffler, C. R. Acad. Sci. Paris 137, 554 (1903).

[43] J. Jadżyn, D. Bauman, J.-L. Déjardin, M. Ginovska, and G. Czechowski, Acta Phys. Pol. 108, 479 (2005). 